\title{
A Self-discharge Model of Lithium-Sulfur Batteries Based on Direct Shuttle Current Measurement
}

Author Names: Vaclav Knap ${ }^{\text {a, }}$, Daniel-Ioan Stroe ${ }^{a}$, Maciej Swierczynski ${ }^{a}$, Rajlakshmi

Purkayastha $^{\mathrm{b}}$, Karsten Propp ${ }^{\mathrm{c}}$, Remus Teodorescu ${ }^{\mathrm{a}}$ and Erik Schaltz ${ }^{\mathrm{a}}$

Affiliation(s): ${ }^{a}$ Department of Energy Technology, Aalborg University, Aalborg, 9000, Denmark

${ }^{\mathrm{b}}$ Oxis Energy Ltd, Culham Science Centre, Abingdon, Oxfordshire OX14 3DB, United Kingdom

${ }^{\mathrm{c}}$ Centre for Automotive Engineering and Technology, Cranfield University, Bedfordshire MK43 0AL, United Kingdom

*Corresponding Author. Tel: +45 20294922, Fax: +45 98151411 E-mail Address:

vkn@et.aau.dk

In the group of post Lithium-ion batteries, Lithium-Sulfur (Li-S) batteries attract a high interest due to their high theoretical limits of the specific capacity of $1672 \mathrm{Ah} \mathrm{kg-1}$ and specific energy of around $2600 \mathrm{Wh} \mathrm{kg}-1$. However, they suffer from polysulfide shuttle, a specific phenomenon of this chemistry, which causes fast capacity fade, low coulombic efficiency, and high self-discharge. The high self-discharge of Li-S batteries is observed in the range of minutes to hours, especially at a high state of charge levels, and makes their use in practical applications and testing a challenging process. A simple but comprehensive mathematical model of the Li-S battery cell self-discharge based on the shuttle current was developed and is presented. The shuttle current values for the model parameterization were obtained from the direct shuttle current measurements. Furthermore, the battery cell depth-of-discharge values were recomputed in order to account for the influence of the self-discharge and provide a higher accuracy of the model. Finally, the derived model was successfully validated against laboratory experiments at various conditions.

Keywords: Lithium-Sulfur battery, self-discharge, polysulfide shuttle, modelling, validation.

\section{Introduction}

Lithium-Sulfur (Li-S) batteries represent a promising alternative to the Lithium-ion battery chemistry, due to their high theoretical limits in terms of specific capacity (i.e. $1672 \mathrm{Ah} \mathrm{kg-1)}$ and specific energy (i.e. $2600 \mathrm{Wh}$ kg-1). Furthermore, they are expected to become a cheaper and more environmentally friendly solution, mainly due to the use of sulfur, which is an abundant and benign element. However, besides other chemistry related phenomena, Li-S batteries suffer from polysulfide shuttle, which results in several commonly known drawbacks: fast capacity fade, low coulombic efficiency, and high self-discharge [1], [2].

For the practical use of the Li-S batteries, there is a need not only to characterize the self-discharge behavior as it was done in [3], but also to provide a proper simulation tool (a model), relevant for industrial applications and laboratory experiments as well; otherwise, biased results can be acquired (e.g. not corresponding depth-of-discharge (DOD) levels assigned). The main cause of self-discharge for Li-S cells was identified to be the polysulfide shuttle and afterwards the corrosion of the current collectors [4], [5], 
[6], [7]. Because the polysulfide shuttle is present not only during the cell idling, but also during charging and discharging, the self-discharge appears as well during these conditions. A mechanistic model of the polysulfide shuttle causing the self-discharge of the Li-S battery cells was presented in [8]. However, the purpose of the model was to provide insights into the key battery mechanisms, rather than to be used from an endapplication perspective. The mathematical model presented in [9] and a zero dimensional model for the Li-S batteries introduced in [10] are using the relations for the polysulfide shuttle derived from [4]. However, these relations are based on determining experimentally a shuttle constant $k_{S}$, which is a time-consuming procedure; moreover, it might not always provide sufficiently accurate results for the self-discharge estimation, as it was indicated in [3]. Another simple approach was used in [11], where the selfdischarge current was related to the charge lost during idling at $100 \%$ state-of-charge (SOC). The self-discharge current was identified to be proportional to the square root of the idling time. However, the model characterization tests for the $100 \%$ SOC condition took more than nine days and it was assumed that self-discharge current is dependent on the used power profile. Furthermore, a methodology for direct shuttle current measurement was proposed in [12], where its results were analyzed and validated using the one-dimensional phenomenological model, which is based on Nernst and species concentrations equations. This methodology allows for a simple and time-effective measurement of the shuttle current at different SOC levels; it is based on the premise that the shuttle current can be observed as the steady-state current flows through the cell, while its voltage is kept constant during constant voltage operation to prevent the voltage decay.

In this paper, the direct shuttle current measurement method is used to identify the shuttle current of a $3.4 \mathrm{Ah} \mathrm{Li-S} \mathrm{pouch} \mathrm{cell} \mathrm{at} \mathrm{different} \mathrm{depth-of-discharge} \mathrm{levels} \mathrm{and}$ temperatures. Furthermore, the obtained results are used to derive a simple and easy-touse mathematical model of the self-discharge in the Li-S battery cell that is related to the polysulfide shuttle phenomenon. This model is validated against several self-discharge experiments at various conditions and it is suitable to predict the self-discharge during idling and operation of the battery.

\section{Methodology}

The work flow followed in this paper is summarized and presented in Fig. 1. At first, the measurements were performed and they are described in Section 2.1 for direct shuttle current measurements and in Section 2.2 for the self-discharge model validation measurements. The current shuttle measurement results are presented in Section 3 and later on in Section 3.1 it is also shown how the mathematical expression for the selfdischarge model dependent on DOD and temperature is derived. Later on, there were considered three fitting cases. Fitting Case 1 (Section 3.1.1) uses the pre-determined DOD points to develop the model, Fitting Case 2 (Section 3.1.2) recomputes and 'corrects' the DOD points according consideration of the self-discharge ongoing during the measurements and Fitting Case 3 (Section 3.1.3) adds up simulation of the measurement to update the DOD points. Each of these fitting cases parameterize the selfdischarge model and its accuracy is later validated in Section 3.2 by an use of the validation measurements (Section 2.2) and the SOC estimation model for the validation (Section 2.3) with the consideration of the total capacity concept (Section 2.4). The discussion about SOC reference frame and cell history effect, which are related to the self-discharge model integration and use, is hold in Section 4.1. Furthermore, the 
alternative version of the self-discharge model considering dependence on the opencircuit voltage rather than DOD is discussed in Section 4.2.

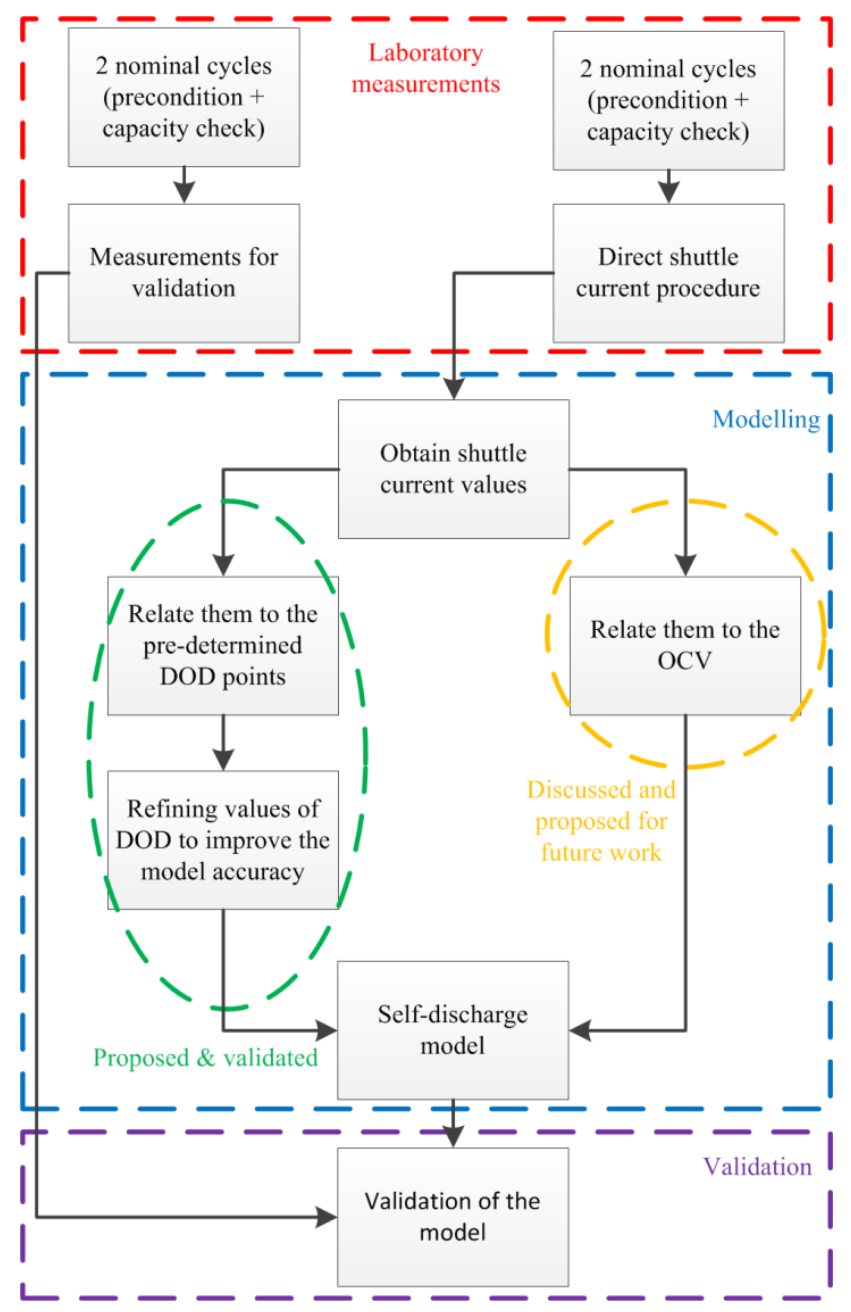

Figure 1. Work flow scheme used for the self-discharge model derivation and validation.

All the measurements were performed on a single 3.4 Ah long life chemistry Li-S pouch cell manufactured by OXIS Energy. A Digatron BTS 600 battery test station was used for the direct shuttle current measurement procedure. To avoid battery cell overcharging and in order to reduce the degradation of the cell at a high current shuttle region, for all the charging conditions, charging time limitations were applied as well (8.5 hours for $15^{\circ} \mathrm{C}, 9$ hours at $25^{\circ} \mathrm{C}$ and 10.5 hours for $35^{\circ} \mathrm{C}$ ). The values of 0.1 and $0.2 \mathrm{C}$ rate correspond to 0.34 and $0.68 \mathrm{~A}$ currents, respectively.

\subsection{Direct Shuttle Current Measurement}

The applied test procedure for the direct shuttle current measurement is based on the methodology presented in [12] and illustrated in Fig. 2. The procedure started with two nominal cycles: 0.1 C-rate constant current charging until $2.45 \mathrm{~V}$ and 0.2 C-rate discharging to $1.5 \mathrm{~V}$. The first cycle served as a pre-condition cycle, which is needed in order to 'reset' the cell's history (as the Li-S is a soluble chemistry) and to bring the cell to the similar initial condition at the selected temperature. The second cycle was used for 
the cell's capacity check and its calculation for the further procedure steps. Afterwards, the cell was charged fully (by $0.1 \mathrm{C}$-rate to $2.45 \mathrm{~V}$ ) and discharged (by $0.2 \mathrm{C}$-rate) to a set DOD point (i.e. $2 \%$ ). Then, the cell was rested in open circuit condition in order to reach an open-circuit voltage (OCV) value. The OCV is considered as an equilibrium voltage point, which is the peak value between voltage rise during the recovery period and voltage fall during the predominant self-discharge. However, in practice, due to the noise in the voltage signal, the reliable value of the OCV was determined when the battery voltage dropped from the maximum point by $0.6 \mathrm{mV}$ (three times the value of the battery test station accuracy which was equal to $0.2 \mathrm{mV}$ ) as it is presented in Fig. 3. In the next step, these detected OCV value was used later in two hours constant voltage charging, in order to determine the steady-state current as it is presented in Fig. 3. This steady-state current is considered as the shuttle current. All these above mentioned steps are repeated for other DOD values (2\% step resolution until $30 \%$ DOD or until there is no detection of the voltage peak in a 12 hours relaxation period). The voltage and current signals during the direct shuttle current measurement procedure are shown in Fig. 3 for DOD equal to $10 \%$ at $35{ }^{\circ} \mathrm{C}$. Next, the direct shuttle current measurement procedure was repeated for other temperatures $\left(15,25\right.$ and $\left.35^{\circ} \mathrm{C}\right)$. The whole measurement procedure, (including the two full cycles performed in the beginning), lasted between 4 and 4.5 days for each of the considered temperatures.

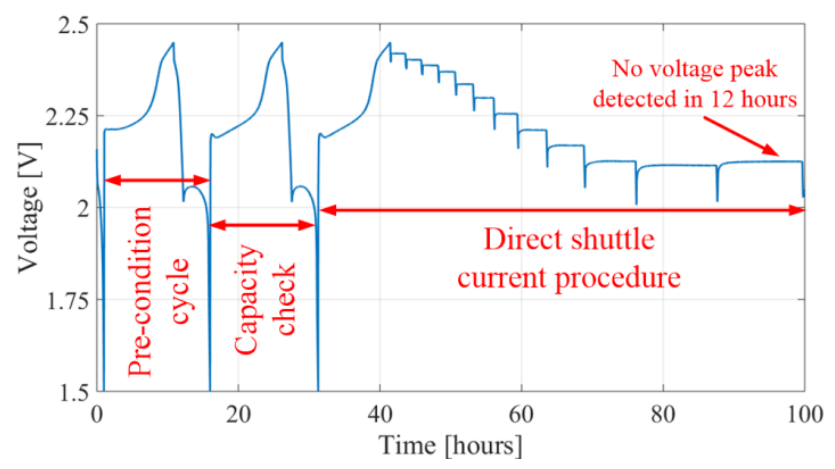

Figure 2. Overall test procedure for the direct shuttle current measurement.
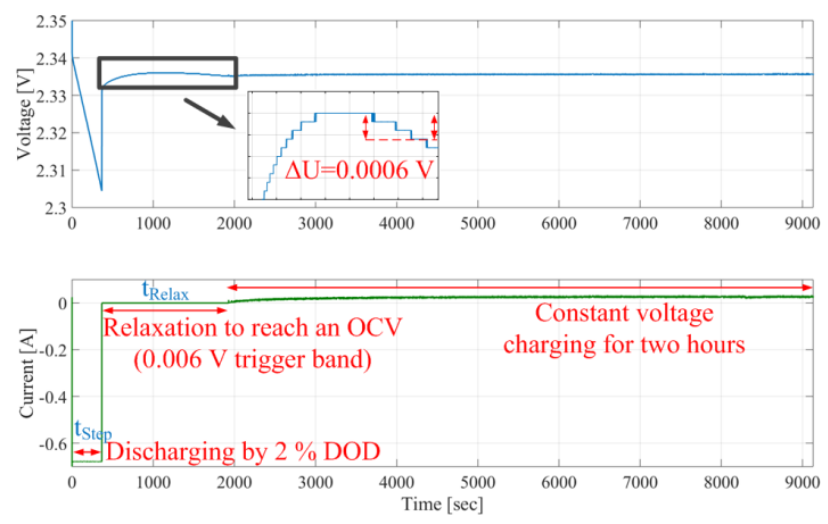

Figure 3. Illustration of current and voltage signals during the direct shuttle current measurement. 
The self-discharge measurement procedure is based on the methodology presented in [3] and it is illustrated in Fig. 4. At first, a pre-condition cycle and capacity check cycle were performed, the same as in the case of the direct shuttle current measurement. This step was followed by charging the cell (by $0.1 \mathrm{C}$-rate to $2.45 \mathrm{~V}$ ) and discharging (by 0.2 C-rate) to a pre-determined DOD value (this discharging step was skipped for $0 \%$ DOD). Then, the cell was kept at open circuit condition ("relaxation stage") for a specific time and afterwards fully discharged (by $0.2 \mathrm{C}$-rate to $1.5 \mathrm{~V}$ ).

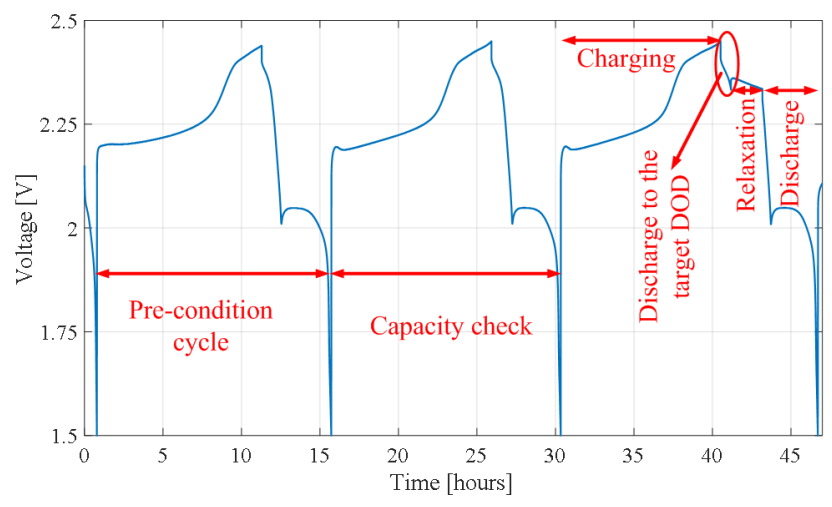

Figure 4. Test procedure for the self-discharge model validation.

\subsection{Matlab/Simulink Model for Validation}

The self-discharge Li-S model is going to be integrated into a Matlab/Simulink model, which allows for SOC estimation based on the coulomb counting method. The used SOC definition in this work follows the definition described in [13]. So the SOC represents the relation between the actual useable battery capacity $\left(C_{a}\right)$ and the total capacity $\left(C_{t}\right)$ available to be discharged after the battery being fully charged. This expressed in percentage is written as $S O C=C_{a} / C_{t}^{*} 100$. Using only coulomb counting method, without accounting for the fast self-discharge of the Li-S batteries will lead inevitably to a growing error due to not capturing the self-discharge current. The SOC estimation model is driven by following equations:

$$
\begin{gathered}
\mathrm{SOC}=\mathrm{SOC}_{\mathrm{ini}}+\int\left(-\left(\mathrm{I}+\mathrm{I}_{\mathrm{sh}}\right) * 100 /\left(\mathrm{C}_{\mathrm{t}} * 3600\right) * \mathrm{dt}\right) \\
\mathrm{DOD}=100-\mathrm{SOC}
\end{gathered}
$$

Where $S O C$ is the actual state-of-charge, $S O C_{i n i}$ is the initial state-of-charge, $I$ is the applied current (discharging current has positive sign orientation), $I_{s h}$ is the shuttle current, $C_{t}$ is the total capacity of the cell at the specific temperature, and $D O D$ is the depth-ofdischarge.

\subsection{Concept of the total capacity of the Li-S batteries}

The standard practice to determine the capacity of Li-S battery cells is to continuously discharge before-hand fully charged battery by a specific current at a specific temperature. The obtained discharged capacity is considered as the capacity of the cell at those conditions. However, as the polysulfide shuttle is present during the Li-S cell discharging, it causes self-discharge, which consequently reduces the measured capacity. Therefore, the term of total capacity $C_{t}$ of the cell is introduced as follows: 


$$
\mathrm{C}_{\mathrm{t}}=\mathrm{C}_{\mathrm{cdch}}+\mathrm{C}_{\mathrm{sd}}
$$

Where $C_{c d c h}$ is the capacity measured during the continuous discharge test for the specific current rate and temperature and $C_{s d}$ is the capacity lost due to the self-discharge during this test. $C_{s d}$ is obtained from the simulation of the cell's continuous discharge with $C_{c d c h}$ replacing $C_{t}$ in (1). Moreover, the $I_{s h}$ is excluded from the coulomb counting in (1) and it is integrated and recorded separately. $C_{s d}$ is the final value of the lost capacity corresponding to the total current $I_{s h}$ recorded during this discharge simulation. This presented concept of the total capacity allows the self-discharge model to estimate the self-discharge during dynamically changing operating conditions.

\section{Measurement Results and Modelling}

The current profiles obtained from the constant voltage charging steps during the direct shuttle current measurements, at $35^{\circ} \mathrm{C}$, are presented in Fig. 5. At least two hours of constant voltage charging are necessary to reach a state close to steady-state. Due to the accuracy of the test station, extra noise is appearing at the current values lower than $0.06 \mathrm{~A}$. In order to get a higher accuracy of the measured shuttle current values, the measurement can be repeated using equipment dedicated for lower current ranges. However, for the demonstration purposes of the model, in this paper, it is considered sufficient to take an average of the last ten minutes of the current profile during constant voltage charging step as the value for the shuttle current. The measured shuttle current values are presented in Fig. 6, for the pre-determined DOD levels.

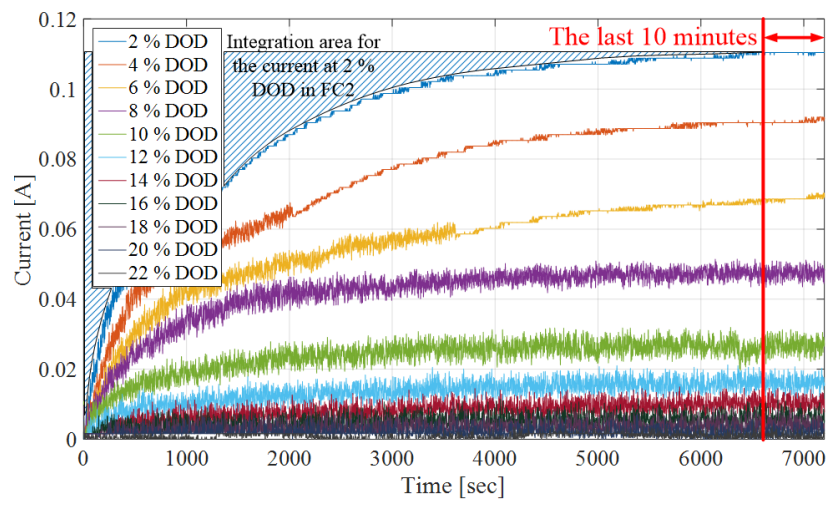

Figure 5. Battery currents during the constant voltage charging step at $35^{\circ} \mathrm{C}$. The shuttle current value is taken as the average of the last ten minutes interval. The integration area for the current at $2 \%$ DOD used in FC2 is marked by blue stripes.

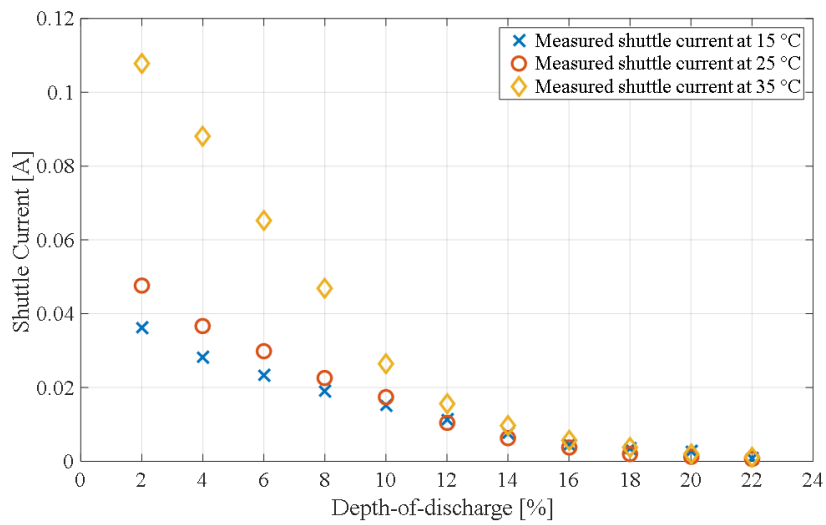


Figure 6. The measured shuttle current values for pre-determined DOD points.

\subsection{Fitting procedure and deriving the model}

Based on the results presented in Fig. 6, it is assumed that an exponential function can be used to describe the dependence of the shuttle current on the DOD. Therefore, all the obtained curves, one for each temperature, are fitted with the following exponential function with parameters $a$ and $b$ :

$$
\mathrm{I}_{\mathrm{sh}}=\mathrm{a} * \exp (\mathrm{b} * \mathrm{DOD})
$$

Furthermore, in a second step, the obtained parameters $a$ and $b$ were fitted as a function of temperature; during this step, it was found out that the dependence on temperature of parameters $\mathrm{a}$ and $\mathrm{b}$ follows an exponential and linear function, respectively. The functions for both parameters $a$ and $b$ are shown below:

$$
\begin{aligned}
& a=c * \exp (d * T e m p) \\
& b=e^{*} \text { Temp }+f
\end{aligned}
$$

By substituting the functions for $a$ and $b$ into (4), the general mathematical model for estimating the self-discharge of Li-S batteries, considering the dependence on DOD and temperature was obtained as given in (7):

$$
\mathrm{I}_{\mathrm{sh}}=\mathrm{c} * \exp (\mathrm{d} * \mathrm{Temp}) * \exp \left(\left(\mathrm{e}^{*} \mathrm{Temp}+\mathrm{f}\right) * \mathrm{DOD}\right)
$$

Where Temp is the temperature in degrees Celsius and $c, d, e$ and $f$ are parameters obtained by fitting the parameters $a$ and $b$ in function of temperature. So, the selfdischarge model's inputs are the temperature and the DOD, while the output is the selfdischarge current, which should be connected to the coulomb counting, if present, or to another implemented SOC estimator.

\subsubsection{Fitting Case 1}

The first fitting step, referred as Fitting Case 1 (FC1), was performed by fitting the experimentally determined direct shuttle current values against the DOD points (see Fig. 6). For the last DOD level, when during the battery cell relaxation period of 12 hours a peak voltage value was not detected (as described in the methodology in the previous section), a shuttle current value equal to zero was considered for fitting purposes. The considered DOD levels are shown in Table I for $\mathrm{FCl}$ and $35^{\circ} \mathrm{C}$. However, these predetermined DOD points might not accurately correspond to the actual DOD levels of the cell as the influence of the shuttle current was not considered during the measurement procedure.

TABLE I. Considered and recomputed DOD values at $35^{\circ} \mathrm{C}$ for fitting at various fitting cases.

\begin{tabular}{ccccccccccccc}
\hline & \multicolumn{1}{c}{ DOD [\%] } \\
\hline FC 1 & 2 & 4 & 6 & 8 & 10 & 12 & 14 & 16 & 18 & 20 & 22 & 24 \\
FC 2 & 3.70 & 7.35 & 10.80 & 13.73 & 16.33 & 18.90 & 21.42 & 23.83 & 26.27 & 28.56 & 30.82 & 32.82 \\
FC 3 & 4.17 & 7.15 & 9.93 & 12.60 & 15.74 & 18.81 & 21.86 & 24.89 & 27.91 & 30.99 & 34.27 & 37.24 \\
\hline
\end{tabular}




\subsubsection{Fitting Case 2}

Therefore, for the Fitting Case 2 (FC2), it was assumed that the self-discharge was ongoing already during the discharging steps, during the relaxation periods before the constant voltage charging step, and during the constant voltage charging step in the characterization experiment, as it is illustrated in Fig. 3. The time values of discharging and relaxation were computed and multiplied by the measured shuttle current value for the first DOD point (i.e. $2 \%$ ), which provided an estimate of the ampere-hours lost due to self-discharge during that period. During two hours of constant voltage charging period (i.e. keeping the constant voltage at the battery terminals), the shuttle current is compensated by an external current approximately in the last ten minutes, as they are considered to be equal there. However, during the previous one hour and fifty minutes, the shuttle current is only partially compensated as the external current is lower. Therefore, the amount of the self-discharged ampere-hours can be obtained by integration of the area above the current curve in a rectangle from the beginning of the constant voltage charging up to one hour and fifty minutes time coordinates. This integration area, as an example for the current at $2 \%$ DOD, is illustrated in Fig. 5 by the blue stripes. The same procedure was repeated for the remaining DOD points, considering also the correction from the previous DOD point. The newly obtained values are presented in Table I. Furthermore, the exact formula describing the correction procedure used for FC2 can be written as:

$$
\begin{array}{r}
D_{\text {new }}(n)=\left(\sum_{1}^{n} D O D_{\text {Step }}(n)+\sum_{1}^{n}\left(t_{\text {step }}(n)+t_{\text {Relax }}(n)\right) \cdot I_{\text {sh }}(n)+\right. \\
\left.\sum_{1}^{n}\left(6600 \cdot I_{\text {sh }}(n)-\int_{0}^{6600} I_{\text {meas }}(n) \cdot d t\right)\right) / C_{c d c h} \cdot \frac{100}{3600}
\end{array}
$$

Where $D O D_{\text {new }}$ is the new recomputed DOD point (replacing a pre-determined DOD point), $n$ is the number of the discharging step, $D O D_{\text {Step }}$ stands for a DOD change to reach the $n$-th pre-determined DOD point from the previous one, $t_{\text {Step }}$ is time spent during the discharging step in seconds, $t_{\text {Relax }}$ is time spent during relaxation before constantvoltage charging step in seconds, $I_{s h}$ is the shuttle current captured for the $n$-th DOD point, $I_{\text {meas }}$ is the measured external current and $C_{c d c h}$ is the capacity of the cell measured during the continuous discharge test in Ampere-hours. 6600 is the number of seconds representing one hour and fifty minutes - after this time the shuttle current is considered to be fully compensated by the external current.

\subsubsection{Fitting Case 3}

For the further improvement of the model, the Fitting Case 3 (FC3) was applied to obtain the total capacity. The Simulink model, including the self-discharge model obtained at the end of FC2, was fed by the current profile obtained from the direct current shuttle procedure. Thus, the DOD points, corresponding to the shuttle current values, were extracted and are presented in Table I for $35^{\circ} \mathrm{C}$.

The parameters $c, d, e$ and $f$ of the shuttle current model for all the fitting cases are presented in Table II.

TABLE II. Found parameters for the fitting cases. 


\begin{tabular}{lcccc}
\hline & $\mathrm{c}$ & $\mathrm{d}$ & $\mathrm{e}$ & $\mathrm{f}$ \\
\hline FC 1 & 0.011000 & 0.07765 & -0.0017110 & -0.07250 \\
FC 2 & 0.009507 & 0.08390 & -0.0009985 & -0.07511 \\
FC 3 & 0.009064 & 0.08709 & -0.0008050 & -0.08524 \\
\hline
\end{tabular}

The presented fitting procedure with all three fitting cases and their steps are visualized in Fig. 7.

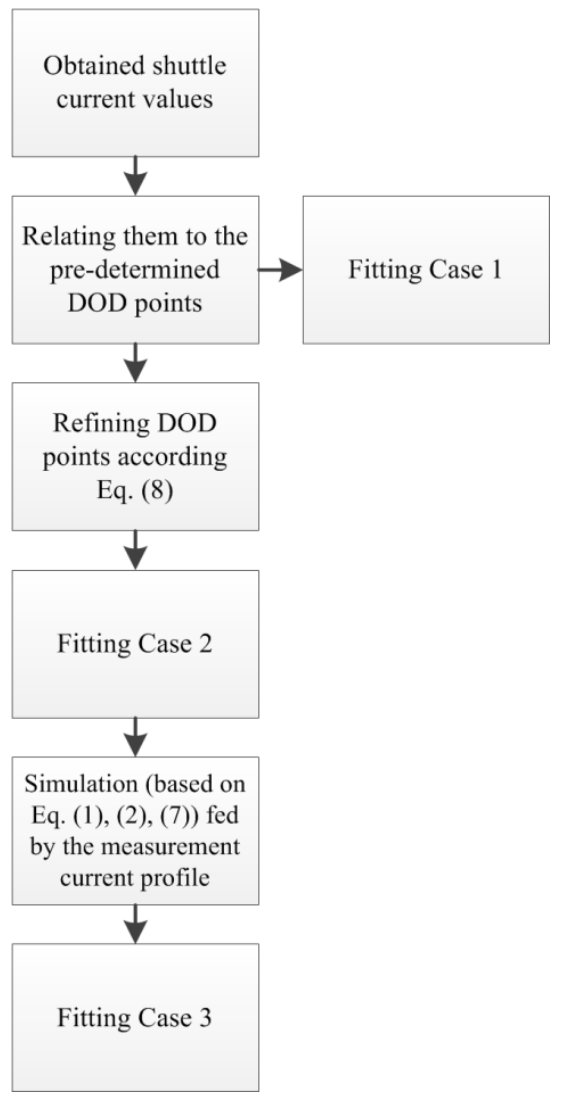

Figure 7. Illustration of the fitting procedure steps.

\subsection{Validation of the self-discharge model}

For the validation of the self-discharge model, four validation measurements were performed according to the procedure described in Section 2.2. The validation cases consider various temperature conditions, idling times and initial DOD levels. The specific conditions are presented in Table III. The absolute self-discharge estimation error $\left(E r r_{a b s}\right)$ and relative self-discharge estimation error $\left(E r r_{r e l}\right)$ were computed as follows:

$$
\begin{gathered}
\text { Err }_{\text {abs }}=\mathrm{C}_{\text {meas }}-\mathrm{C}_{\text {est }} \\
\operatorname{Err}_{\text {rel }}=\left(\mathrm{C}_{\text {meas }}-\mathrm{C}_{\text {est }}\right) / \mathrm{C}_{\text {meas }} * 100
\end{gathered}
$$

Where $C_{\text {meas }}$ is remaining measured capacity and $C_{\text {est }}$ is remaining estimated capacity.

A comparison of the accuracy values of the developed self-discharge model for the different used fitting cases is shown in Table III. The relative errors are noticeably reduced by moving from $\mathrm{FC} 1$ to $\mathrm{FC} 2$, except the Validation Case 1, where only a minor increase is observed. The error reduction implies the correctness of the assumptions used 
for FC2 and that the self-discharge due to the polysulfide shuttle is still present, no matter if the Li-S battery is in charging, relaxation or discharging stage. The further move to FC3 did not bring any improvements in terms of accuracy. However, this might be related to the fitting of the parameter $b$, which in the FC3 lost its linear character and thus the fitting error was increased. To confidently determine the new trend, it might be needed to have more temperature points available for the fitting.

TABLE III. Relative errors of validation cases for different fitting cases.

\begin{tabular}{|c|c|c|c|}
\hline Validation cases & & Relative error & \\
\hline & Fitting Case 1 & Fitting Case 2 & Fitting Case 3 \\
\hline Validation Case 1 & $1.29 \%$ & $1.60 \%$ & $1.59 \%$ \\
\hline $\begin{array}{l}0 \% \text { DOD, } 20{ }^{\circ} \mathrm{C}, 4 \text { hours idling } \\
\text { Validation Case } 2 \\
0 \% \text { DOD, } 30{ }^{\circ} \mathrm{C}, 12 \text { hours idling }\end{array}$ & $11.04 \%$ & $6.65 \%$ & $6.69 \%$ \\
\hline $\begin{array}{c}\text { Validation Case } 3 \\
10 \% \text { DOD, } 25^{\circ} \mathrm{C}, 6 \text { hours idling }\end{array}$ & $4.62 \%$ & $2.66 \%$ & $2.82 \%$ \\
\hline $\begin{array}{c}\text { Validation Case } 4 \\
15 \% \text { DOD }, 35^{\circ} \mathrm{C}, 2 \text { hours idling }\end{array}$ & $7.98 \%$ & $3.95 \%$ & $3.96 \%$ \\
\hline
\end{tabular}

The detailed results for the validation of the proposed self-discharge model, which was parametrized based on FC2, are shown in Table IV. One can observe that the model is capable to predict accurately the self-discharge ongoing in Li-S batteries, for short term (e.g., less than twelve hours) with a relative error smaller than $7 \%$; furthermore, for most real-life applications relaxation periods longer than 12 hours are not very common. This model is applicable for the temperatures inside the characterization window between 15 and $35{ }^{\circ} \mathrm{C}$, according to the performed experiments. It is worth to note that the selfdischarge estimation error is influenced also by the irreversible degradation, appeared during the characterization and verification experiments. By accounting for this degradation, the model error can be further decreased. Moreover, because the Li-S is a solution chemistry, changes in the polysulfide species might occur, accelerated by longer time span and higher temperature, which could further negatively influence the final accuracy of the model.

TABLE IV. Measured and estimated battery cell capacities and their absolute and relative errors for four validation cases with the self-discharge model parametrized based on FC2.

\begin{tabular}{|c|c|c|c|c|}
\hline Validation cases & $\begin{array}{c}\text { Final capacity } \\
\text { measured }\end{array}$ & $\begin{array}{c}\text { Final capacity } \\
\text { estimated }\end{array}$ & $\begin{array}{c}\text { Absolute } \\
\text { error }\end{array}$ & Relative error \\
\hline $\begin{array}{c}\text { Validation Case } 1 \\
0 \% \text { DOD }, 20^{\circ} \mathrm{C}, 4 \text { hours idling }\end{array}$ & $2.606 \mathrm{Ah}$ & $2.5642 \mathrm{Ah}$ & $0.0418 \mathrm{Ah}$ & $1.60 \%$ \\
\hline $\begin{array}{c}\text { Validation Case } 2 \\
0 \% \text { DOD, } 30{ }^{\circ} \mathrm{C}, 12 \text { hours idling }\end{array}$ & $2.262 \mathrm{Ah}$ & $2.4125 \mathrm{Ah}$ & $-0.1505 \mathrm{Ah}$ & $6.65 \%$ \\
\hline $\begin{array}{c}\text { Validation Case } 3 \\
10 \% \text { DOD, } 25^{\circ} \mathrm{C}, 6 \text { hours idling }\end{array}$ & $2.273 \mathrm{Ah}$ & $2.3335 \mathrm{Ah}$ & $-0.0605 \mathrm{Ah}$ & $2.66 \%$ \\
\hline $\begin{array}{c}\text { Validation Case } 4 \\
15 \% \text { DOD, } 35^{\circ} \mathrm{C}, 2 \text { hours idling }\end{array}$ & $2.399 \mathrm{Ah}$ & $2.4937 \mathrm{Ah}$ & $-0.0947 \mathrm{Ah}$ & $3.95 \%$ \\
\hline
\end{tabular}

\section{Discussion}

\subsection{SOC reference frame \& cell history effect}

The challenging part of the integration of the presented self-discharge model into any other model is that the battery performance model has to have the same DOD/SOC reference frame in order that the dependency states to be matched. Due to the "rate 
capacity effect' [14], the available battery capacity varies according the applied current. Therefore, $\mathrm{C}_{\mathrm{cdch}}$ term is also current dependent. Usually, to obtain this $\mathrm{C}_{\mathrm{cdch}}$, continuous discharge tests are used. However, alternative approaches can be followed. In [15], for practical reasons, the mixed pulse discharge was used to determine $\mathrm{C}_{\mathrm{cdch}}$. This procedure combined three different currents interleaved with the relaxation periods, which means the different charge and discharge protocols. Therefore, the DOD of the performance model has to be converted into DOD of the self-discharge model at its input. Alternatively, the self-discharge model should be parametrized by performing direct current shuttle measurement already in the target DOD reference frame, which is used in the performance model. The DOD definition of the presented self-discharge model is based on the continuous discharge capacity by a $0.2 \mathrm{C}$-rate. Moreover, this self-discharge takes place mainly in high voltage plateau; therefore if the capacity of the performance model is divided into high- and low-voltage plateau, only the high-voltage plateau capacity should be updated in the concept of the total capacity.

Another feature of the Li-S batteries is that they are a solution based chemistry, so the previous history of the cell (cycling/storage at specific conditions) influences its current performance. This feature has not been so far properly addressed at a sufficiently simplified level in order to be used for battery management systems and practical applications. Therefore our work, similar to [3], uses a pre-condition cycle as a part of the test procedure (charge and discharge protocol) in order to 'reset the memory' and to reach repeatable results.

\subsection{Open-circuit voltage based self-discharge model}

Alternatively, the DOD dependence of the self-discharge model can be replaced by the open-circuit voltage (OCV) dependence. By following this approach, the shuttle current values related to the OCV are presented in Fig. 8, where it is important to note that this relation is valid only for OCV values at the side of the high voltage plateau. The OCV values in the practical use can be obtained for example by online parameter identification techniques [16]. The use of the identification techniques is required because of even though the OCV is directly measurable at the cell relaxed for a sufficient long time, this condition might be difficult to reach during the operation. Thus, the control system should decide what is the actual OCV value to be used as the input for the selfdischarge model.

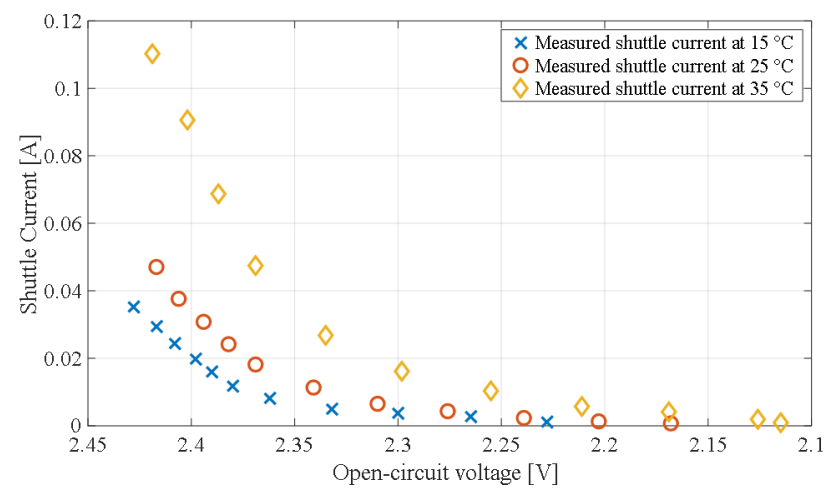

Figure 8 . The measured shuttle current values related to the open-circuit voltage values (at the side of the high voltage plateau).

\section{Conclusions}


The direct shuttle current measurement methodology was applied to a $3.4 \mathrm{Ah} \mathrm{Li-S}$ pouch cell, which allows for the shuttle current quantification at various battery cell DODs and temperatures. In this study, the shuttle current is considered as the only source of the self-discharge and therefore, the high voltage plateau was in focus. The predetermined DOD steps from the measurement were recomputed in order to take into account the shuttle current and thus obtain the actual corresponding DOD levels. Based on the curve fitting of the shuttle current dependency on DOD and temperature, a simple mathematical model for the self-discharge estimation of Li-S batteries was obtained. This model is dedicated to the estimation of the short-term self-discharge in the range of hours. The self-discharge of Li-S batteries is considerably higher and faster in comparison to other battery technologies and it is caused by the shuttle current and appears mainly at low DOD states. The developed model was successfully validated by the experiments considering various conditions with a relative error smaller than $7 \%$. Therefore, this model represents a powerful tool for the self-discharge estimation of the Li-S batteries. Due to its simplicity and low computational demand, it is suitable to be integrated into battery management systems. Moreover, it can be used also as a part of SOC estimation, which is a challenging task for this particular battery chemistry. Additionally, the concept of the total capacity for Li-S batteries is proposed in order to account for the selfdischarge during the operation of the cell. Furthermore, the self-discharge model use can prevent a bias at analyzing experimental results with wrongly determined DOD values of the cell.

\section{Acknowledgments}

This work has been part of the ACEMU-project. The authors gratefully acknowledge the Danish Council for Strategic Research (1313-00004B) and EUDP (1440-0007) for providing financial support and would like to thank OXIS Energy for supplying the Lithium-Sulfur battery cells.

\section{References}

[1] D. Bresser, S. Passerini, B. Scrosati, Recent progress and remaining challenges in sulfur-based lithium secondary batteries - a review, Chem. Commun. 49 (2013) 10545-10562. doi:10.1039/c3cc46131a.

[2] M. Wild, L. O’Neill, T. Zhang, R. Purkayastha, G. Minton, M. Marinescu, G.J. Offer, Lithium Sulfur Batteries, A Mechanistic Review, Energy Environ. Sci. (2015). doi:10.1039/C5EE01388G.

[3] V. Knap, D.-I. Stroe, M. Swierczynski, R. Teodorescu, E. Schaltz, Investigation of the Self-Discharge Behavior of Lithium-Sulfur Batteries, J. Electrochem. Soc. 163 (2016) A911-A916. doi:10.1149/2.0641606jes.

[4] Y. V. Mikhaylik, J.R. Akridge, Polysulfide Shuttle Study in the Li/S Battery System, J. Electrochem. Soc. 151 (2004) A1969. doi:10.1149/1.1806394.

[5] H. Ryu, H. Ahn, K. Kim, J. Ahn, J. Lee, E. Cairns, Self-discharge of lithiumsulfur cells using stainless-steel current-collectors, J. Power Sources. 140 (2005) 365-369. doi:10.1016/j.jpowsour.2004.08.039.

[6] H.S. Ryu, H.J. Ahn, K.W. Kim, J.H. Ahn, K.K. Cho, T.H. Nam, Self-discharge characteristics of lithium/sulfur batteries using TEGDME liquid electrolyte, Electrochim. Acta. 52 (2006) 1563-1566. doi:10.1016/j.electacta.2006.01.086.

[7] M. Kazazi, M.R. Vaezi, A. Kazemzadeh, Improving the self-discharge behavior of sulfur-polypyrrole cathode material by LiNO3 electrolyte additive, Ionics (Kiel). 
20 (2014) 1291-1300. doi:10.1007/s11581-014-1095-2.

[8] A.F. Hofmann, D.N. Fronczek, W.G. Bessler, Mechanistic modeling of polysulfide shuttle and capacity loss in lithium-sulfur batteries, J. Power Sources. 259 (2014) 300-310. doi:10.1016/j.jpowsour.2014.02.082.

[9] M.R. Busche, P. Adelhelm, H. Sommer, H. Schneider, K. Leitner, J. Janek, Systematical electrochemical study on the parasitic shuttle-effect in lithium-sulfurcells at different temperatures and different rates, J. Power Sources. 259 (2014) 289-299. doi:10.1016/j.jpowsour.2014.02.075.

[10] M. Marinescu, T. Zhang, G.J. Offer, A zero dimensional model of lithium-sulfur batteries during charge and discharge, Phys. Chem. Chem. Phys. (2015). doi: $10.1039 / \mathrm{C} 5 \mathrm{CP} 05755 \mathrm{H}$.

[11] C.E. Parfitt, Characterisation, Modelling and Management of Lithium Sulphur Batteries for Spacecraft Applications, University of Warwick, 2012.

[12] D. Moy, a. Manivannan, S.R. Narayanan, Direct Measurement of Polysulfide Shuttle Current: A Window into Understanding the Performance of Lithium-Sulfur Cells, J. Electrochem. Soc. 162 (2014) A1-A7. doi:10.1149/2.0181501jes.

[13] W. Waag, C. Fleischer, D.U. Sauer, Critical review of the methods for monitoring of lithium-ion batteries in electric and hybrid vehicles, J. Power Sources. 258 (2014) 321-339. doi:10.1016/j.jpowsour.2014.02.064.

[14] M. Doyle, J. Newman, Analysis of capacity-rate data for lithium batteries using simplified models of the discharge process, J. Appl. Electrochem. 27 (1997) 846856. doi:10.1023/A:1018481030499.

[15] K. Propp, M. Marinescu, D.J. Auger, L. O’Neill, A. Fotouhi, K. Somasundaram, G.J. Offer, G. Minton, S. Longo, M. Wild, V. Knap, Multi-temperature statedependent equivalent circuit discharge model for lithium-sulfur batteries, J. Power Sources. 328 (2016) 289-299. doi:10.1016/j.jpowsour.2016.07.090.

[16] A. Fotouhi, D.J. Auger, K. Propp, S. Longo, A Study on Battery Model Parametrisation Problem - Application-Oriented Trade-offs between Accuracy and Simplicity, in: 8th IFAC Symp. Adv. Automot. Control (AAC 2016), 2016: pp. 19-23. 
2016-10-29

\section{A self-discharge model of Lithium-Sulfur batteries based on direct shuttle current measurem}

Knap, Vaclav

Elsevier

Knap V, Stroe D-I, Swierczynski M, Purkayastha R, Propp K, Teodorescu R, Schaltz E, A self-discharge model of Lithium-Sulfur batteries based on direct shuttle current measurement, pÿJournal of Power Sources, Volume 336, 30 December 2016, Pages 325331

http://dx.doi.org/10.1016/j.jpowsour.2016.10.087

Downloaded from Cranfield Library Services E-Repository 